\title{
Diagnostic Utility of Transbronchial Needle Aspiration in Malignant Endobronchial Lesions: Relevance to Lesions' Characteristics
}

\author{
Sherif A. A. Mohamed, ${ }^{1}$ Yousef Ahmed, ${ }^{1}$ Khaled Hussein, ${ }^{1}$ \\ Nashwa M. A. Abd El-Aziz, ${ }^{2}$ and Yasser Gamal ${ }^{3}$ \\ ${ }^{1}$ Department of Chest Diseases, Faculty of Medicine, Assiut University, Assiut 71516, Egypt \\ ${ }^{2}$ Department of Medical Oncology, South Egypt Cancer Institute, Assiut University, Assiut 71516, Egypt \\ ${ }^{3}$ Department of Pathology, Faculty of Medicine, Assiut University, Assiut 71516, Egypt
}

Correspondence should be addressed to Sherif A. A. Mohamed; saawm20@yahoo.com

Received 27 June 2013; Revised 9 August 2013; Accepted 22 August 2013

Academic Editor: Akira Mogi

Copyright (C) 2013 Sherif A. A. Mohamed et al. This is an open access article distributed under the Creative Commons Attribution License, which permits unrestricted use, distribution, and reproduction in any medium, provided the original work is properly cited.

In this prospective study, we aimed to report our experience with the diagnostic utility of transbronchial needle aspiration (TBNA) in patients with malignant endobronchial lesions detected during routine bronchoscopy. Ninety-four patients were enrolled. TBNA and conventional diagnostic techniques (CDTs: forceps biopsy, brushing, and washing) were performed in all patients. Endobronchial lesions were classified into exophytic mass lesions (EMLs), submucosal disease (SD), and peribronchial disease (PD). The diagnostic yields of TBNA and CDT alone and together were compared according to the lesions' types, histopathology, and locations. During 3-year period, the addition of TBNA to CDT improved bronchoscopic sensitivity from $70.2 \%$ to $94.7 \%$ in all lesion types. Addition of TBNA to CDT increased the diagnostic success from $74 \%$ to $95 \%$ and from $50 \%$ to $94 \%$ in NSCLC and SCLC, respectively. The diagnostic success was increased in all localizations by the addition of TBNA to CDT, particularly for lesions located at the trachea, main bronchi, and upper lobes. We conclude that the addition of TBNA to CDT increases the diagnostic yield in patients with visible malignant endobronchial lesions, particularly in peribronchial disease, and improves the diagnostic yield of bronchoscopy, in both NSCLC and SCLC and in all bronchoscopic locations, particularly in central and upper lobar lesions.

\section{Introduction}

Transbronchial needle aspiration (TBNA) is a modality that allows the bronchoscopist to sample tissue from the deeper submucosa as well as from the close extraluminal areas of the endobronchial tree. TBNA is a beneficial, safe, and minimally invasive technique that was proved to be useful in the diagnosis and staging of lung cancer [1]. Previously, the utility of TBNA was restricted to mediastinal lymph node and extrabronchial lesion sampling. Its use has been expanded to complement conventional diagnostic techniques (CDTs) such as bronchial washing (BW), bronchial brushing (BB), and forceps biopsy (FB) in the diagnosis of endobronchial lung cancer [2]. However, only few studies [27] had addressed the diagnostic utility of TBNA in visible endobronchial lung cancer. Moreover, despite that TBNA proved cost-effective diagnostic utility in visible malignant endobronchial lesions [8], studies evaluating that utility in developing countries are still lacking. Lung cancer may present either as a parenchymal lesion or as endobronchial disease. The latter may manifest as an exophytic mass lesion (EML), submucosal infiltration (submucosal disease, SD), or extrinsic compression from peribronchial disease (PD) $[1,2]$. The impact of different characteristics of endobronchial lesions (e.g., type, histopathologic subtype, location) on the diagnostic utility of TBNA needs to be elucidated $[2,7]$. The aims of this study were; first, to report our experience with the diagnostic utility of TBNA and its contributions to $\mathrm{CDT}$ in visible endobronchial lesions of the patients who underwent TBNA during fiberoptic bronchoscopy (FOB) and 
had the final diagnosis of primary lung cancer and Second, to evaluate this utility in relation to different endobronchial lesions' characteristics (i.e., type, histopathologic subtype, and location).

\section{Patients and Methods}

Assiut University Hospital is a large tertiary hospital in Upper Egypt, to which many patients with suspected or known lung cancer are referred. Patients who had the preliminary diagnosis of lung cancer in whom EML, SD, or PD was detected by routine FOB underwent TBNA in addition to the bronchoscopic CDT and were prospectively enrolled from January, 2010 to December, 2012 into this study. Patients with preexisting known malignancy were excluded. Submucosal disease was defined as erythema, vascular flares, enhanced rugal pattern, thickening or loss of mucosal markings, and bronchial narrowing [1, 2, 7]. Peribronchial disease was defined as luminal narrowing secondary to extrinsic compression $[1,2]$. Lesions located in trachea and in main and lobar bronchi were classified as "central," and those with segmental and subsegmental localization were classified as "peripheral" [6]. All patients underwent all procedures (CDT and TBNA). The sequence was always washing, TBNA, forceps biopsy, and brushing to minimize specimen contamination and avoid false-positive results.

Prior to bronchoscopy, all patients underwent chest radiography and computed tomography (CT) of the chest. Complete blood count and coagulation tests (bleedingaggregation and prothrombin time) were performed. Patients fasted overnight and were given $10 \mathrm{mg}$ of diazepam and $0.5 \mathrm{mg}$ of atropine intramuscularly as premedication $30 \mathrm{~min}$ prior to the examination. Local anesthesia was performed with a lidocaine solution. A flexible fiberoptic bronchoscopy (Pentax SB 15; Pentax, Japan) under local anaesthesia was inserted via nasal or oral route with the patient lying in supine position. A $13 \mathrm{~mm}$ 21-gauge cytology needle (NA-2C1; Olympus Corporation) was used for TBNA. The transbronchial needle was introduced into the bronchoscope with the needle inside the metal hub while visualizing the trachea through the scope. When the metal hub was visible, the needle was advanced. In the presence of PD, the needle was inserted into the lesion according to the "pushing" technique [9]. In EML, the needle was penetrated into the mass directly. In patients with $\mathrm{SD}$, the needle was inserted at a $45^{\circ}$ angle into the puncture site. One cytologic sample consisted of at least three subsequent aspirations from the same region, penetrating the bronchial wall in an area $<2 \mathrm{~cm}^{2}$. A $50 \mathrm{~mL}$ syringe was used to apply suction $[6,7]$, and it was detached before retraction of the needle in order to avoid contamination. The aspirated material was blown into four or five slides, smeared, immediately fixed with $95 \%$ alcohol, and sent for cytologic examination. After the TBNA procedure, $\mathrm{FB}$ and $\mathrm{BB}$ were performed in a conventional manner. Four adequate FB specimens were performed and then fixed in formaldehyde solution. Histologic specimens were stained with hematoxylin-eosin, and cytologic specimens were stained by the Papanicolaou technique. On-site cytopathologic assessment was not performed. All specimens
TABLE 1: Diagnostic yield of TBNA, CDT, and CDT plus TBNA with respect to the type of lesions*.

\begin{tabular}{lcccc}
\hline Condition & $\begin{array}{c}\text { TBNA } \\
\text { no. }(\%)\end{array}$ & $\begin{array}{c}\text { CDT } \\
\text { no. }(\%)\end{array}$ & $\begin{array}{c}\text { CDT + TBNA } \\
\text { no. }(\%)\end{array}$ & $P$ value \\
\hline EML $(n=34)$ & $27(79.4)$ & $28(82.3)$ & $33(97)$ & 0.063 \\
SD $(n=23)$ & $21(91)$ & $17(74)$ & $22(95.6)$ & 0.063 \\
PD $(n=37)$ & $34(92)$ & $21(57)$ & $34(92)$ & $<0.001$ \\
SPD $(n=60)$ & $55(91.7)$ & $38(63.3)$ & $56(93.3)$ & $<0.001$ \\
Total $(n=94)$ & $82(87.2)$ & $66(70.2)$ & $89(94.7)$ & $<0.001$ \\
\hline
\end{tabular}

${ }^{*}$ CDT versus CDT + TBNA; EML: exophytic mass lesions; SD: submucosal disease; PD: peribronchial disease; SPD: submucosal-peribronchial disease.

were interpreted by one cytopathologist. Results of cytologic analysis were considered positive only when a sufficient number of definitely malignant cells were observed. All specimens having cellular atypia and abnormal cells highly suggestive of malignancy were considered negative. The primary outcome to measure was to evaluate the diagnostic yield from TBNA, CDT, and TBNA plus CDT; yields of CDT were compared to those of CDT plus TBNA. We also studied and compared the diagnostic yields from individual procedures and questioned for the best yield of their combination(s). The secondary outcome was to address the diagnostic yield of TBNA and its contribution to CDT in relation to the type of the endobronchial lesion (i.e., EML, SD, or PD), histologic subtypes, and location. Any procedure-related complications or damage to the bronchoscope were documented. The study was approved by the local ethical committee, and a written consent was obtained from every patient for enrollment into this study.

Data were analysed using the statistical package for the Social Sciences (Windows version 16.0; SPSS Inc., Chicago, IL, USA). Statistical analysis of the difference in diagnostic yield between the two groups was performed using $\mathrm{McNe}$ mar's test. Fisher's exact test was used to detect any difference between locations. A $P$ value $<0.05$ was considered to be significant.

\section{Results}

During a 3-year period, 94 patients had bronchoscopically visible endobronchial lesions. Samples of TBNA, forceps biopsy, brushing, and washing were collected from all patients. With these diagnostic methods, 89 patients $(94.7 \%)$ had a diagnosis of primary lung cancer, but in 5 patients, no diagnosis was made. These five patients were diagnosed by other techniques (pleural biopsy, CT-guided biopsy, and transthoracic needle biopsy). The patients included 81 (86\%) men and $13(14 \%)$ women, with a mean age of $58.39 \pm 6.14$ years and range of 42-78 years. Histopathologic diagnosis was nonsmall cell lung cancer (NSCLC) in 78 (83\%) patients and small cell lung cancer (SCLC) in 16 (17\%) patients. Bronchoscopically, EML, SD, and PD were encountered in $34 / 94,36 \%, 23 / 94,24 \%$, and $37 / 94,40 \%$ of patients, respectively. Diagnostic yield of TBNA, CDT, and CDT plus TBNA is shown in Table 1. Diagnosis was made by CDT in 66 of 94 patients $(70.2 \%)$. The addition of TBNA to CDT enabled 
histopathologic diagnosis in another 23 patients $(24.5 \%)$ and improved the sensitivity of bronchoscopy from 70.2 to $94.7 \%$ $(P<0.001)$. The sensitivities of CDT, TBNA, and CDT plus TBNA were compared for different endobronchial lesion types. The addition of TBNA to CDT increased sensitivity in all lesion types, and this increase was statistically significant in the peribronchial disease group $(P<0.001)$. In this group, diagnosis was made solely by TBNA in 13 patients $(35 \%$ of the PD group). When the 60 submucosal and peribronchial diseases (SPDs) were considered together, the addition of TBNA to CDT increased the diagnostic yield from 63 to 93\%, and the difference was highly significant $(P<0.001)$. Despite that no statistical difference was observed in the EML group, TBNA identified five additional patients with lung cancer. Table 2 demonstrates the different diagnostic yields from individual procedures and their combination. Rate of diagnostic yield was the highest for TBNA (82/94 patients, $87.2 \%$ ), followed by forceps biopsy (52/94 patients, $55.3 \%)$, brushing (49/94 patients, 52\%), and washing (24/94 patients, $25.5 \%$ ). The rate of diagnostic yield was also the highest for TBNA in both EML and SPD groups, 79.4\% and $91.7 \%$, respectively. Interestingly, diagnostic yield from TBNA was far superior to the yield from combination of forceps biopsy and brushing in total lesions and SPD (87.2\% versus $70.2 \%$ and $91.7 \%$ versus $63.3 \%$, resp.). Combination of TBNA with forceps and brushing provided the best diagnostic yield, 97\%, 93.3\%, and 94.7\%, in EML, SPD, and total lesions, respectively.

TBNA was exclusively diagnostic in 13 patients $(14 \%$ of all patients): 9 patients with SPD (15\% of patients with SPD) and 4 patients with EML (15\% of patients with EML). Among those 4 patients with EML, there were two patients with small cell carcinoma who had crush artifacts on the FB and were solely diagnosed by TBNA. Forceps biopsy and brushing were exclusively diagnostic in six (6\%) and three $(3 \%)$ of patients; respectively, and all these patients had EML. Bronchial washing was not exclusively diagnostic in situations in which all other procedures were negative. Comparing the diagnostic yield of TBNA and forceps biopsy for all types of endobronchial lesions revealed a highly significant statistical difference $(82 / 94,87.2 \%$ versus $52 / 94$, 55.3\%; $P<0.001$ ). (Figure 1) This difference was primarily due to difference in the SPD group (55/60, 91.7\% versus 26/60, 43\%; $P<0.001)$. The diagnostic yield of TBNA with respect to histologic subtypes is demonstrated in Table 3. An increment from 74.4 to $95 \%$ was observed in diagnostic yield in NSCLC when TBNA was combined with CDT $(P<0.001)$. The diagnostic efficacy improved from 58.8 to $91.2 \%$ by the addition of TBNA to CDT in the peribronchial disease group for NSCLC $(P<0.001)$. However, the addition of TBNA to CDT did not result in a statistically significant difference in EML and SD groups for NSCLC. In the SCLC group, the diagnosis was made by CDT in 8 of 16 patients (50\%), and the diagnosis was made by CDT plus TBNA in 15 patients (94\%) $(P=0.016)$. However, when the differences between CDT and CDT plus TBNA were analysed in different types of lesions within the SCLC group, there was no significant statistical difference for any of these lesions (60\% versus $100 \%$, $50 \%$ versus $87.5 \%$, and $33.3 \%$ versus $100 \%$ for $\mathrm{EML}$,
TABLE 2: Diagnostic yield of individual procedures and their combinations*.

\begin{tabular}{lcccc}
\hline Procedure & $\begin{array}{c}\text { EML } \\
(n=34)\end{array}$ & $\begin{array}{c}\text { SPD } \\
(n=60)\end{array}$ & $\begin{array}{c}\text { Total } \\
(n=94)\end{array}$ & $\begin{array}{c}\text { Sole } \\
\text { diagnostic } \\
\text { technique }\end{array}$ \\
\hline BW & $10(29.4)$ & $14(23.3)$ & $24(25.5)$ & $0(0)$ \\
BB & $21(61.8)$ & $28(50)$ & $49(52)$ & $3(3)$ \\
FB & $26(76.5)$ & $26(43)$ & $52(55.3)$ & $6(6)$ \\
TBNA & $27(79.4)$ & $55(91.7)$ & $82(87.2)$ & $13(14)$ \\
FB + BB & $28(82.3)$ & $38(63.3)$ & $66(70.2)$ & \\
FB + TBNA & $27(79.4)$ & $55(91.7)$ & $82(87.2)$ & \\
FB + BB + TBNA & $33(97)$ & $56(93.3)$ & $89(94.7)$ & \\
\hline
\end{tabular}

${ }^{*}$ Values given as no. (\%) BW: bronchial washing; BB: bronchial brushing; FB: forceps biopsy; TBNA: transbronchial needle aspiration.

TABLE 3: Diagnostic yield of TBNA, CDT, and CDT plus TBNA with respect to the histopathologic subtype*.

\begin{tabular}{lcccc}
\hline $\begin{array}{l}\text { Histopathologic } \\
\text { subtype/lesion }\end{array}$ & $\begin{array}{c}\text { TBNA } \\
\text { no. }(\%)\end{array}$ & $\begin{array}{c}\text { CDT } \\
\text { no. }(\%)\end{array}$ & $\begin{array}{c}\text { CDT + TBNA } \\
\text { no. }(\%)\end{array}$ & $P$ value \\
\hline NSCLC $(n=78)$ & & & & \\
EML $(n=29)$ & $24(82.8)$ & $25(86.2)$ & $28(96.6)$ & 0.25 \\
SD $(n=15)$ & $15(100)$ & $13(86.7)$ & $15(100)$ & 0.5 \\
PD $(n=34)$ & $31(91.2)$ & $20(58.8)$ & $31(91.2)$ & 0.001 \\
\hline Total $(n=78)$ & $70(90)$ & $58(74.4)$ & $74(95)$ & $<0.001$ \\
\hline SCLC $(n=16)$ & & & & \\
EML $(n=5)$ & $3(60)$ & $3(60)$ & $5(100)$ & 0.25 \\
SD $(n=8)$ & $6(75)$ & $4(50)$ & $7(87.5)$ & 0.125 \\
PD $(n=3)$ & $3(100)$ & $1(33.3)$ & $3(100)$ & 0.125 \\
\hline Total $(n=16)$ & $12(75)$ & $8(50)$ & $15(94)$ & 0.016 \\
\hline
\end{tabular}

${ }^{*}$ CDT versus TBNA + CDT; NSCLC: nonsmall cell lung cancer; SCLC: small cell lung cancer.

$\mathrm{SD}$, and PD, resp.). The diagnostic utility of CDT and CDT plus TBNA according to the location of endobronchial lesions was addressed (Table 4). The lesions were in the trachea or main bronchi in 27 patients (29\%), in upper lobes in 46 patients (49\%), in the right middle lobe in 9 patients $(9.3 \%)$, and in the lower lobes in 12 patients $(12.7 \%)$. The addition of TBNA to CDT increased the diagnostic yield irrespective of the location of the lesion, and this increase was significant in trachea and main bronchi and bronchi of the upper lobes. When the right and left upper lobes were compared, this significance was attributed to right upper lobe lesions rather than left upper lobe ones. Rightor left-sided malignant lesions demonstrated no difference due to diagnostic efficacy. Also, no statistically significant difference was observed among the diagnostic yield of each method with regard to the lesion being central or peripheral. Results from distribution of malignant cell types obtained by cytologic assessment from TBNA were in complete concordance with those obtained by histologic assessment by forceps biopsy. Severe complications such as major bleeding and pneumothorax were not observed. Minor bleeding, 
TABLE 4: Diagnostic yield of TBNA, CDT, and CDT plus TBNA with respect to localization*.

\begin{tabular}{|c|c|c|c|c|}
\hline Localization & TBNA no. $(\%)$ & CDT no. (\%) & CDT + TBNA no. (\%) & $P$ value \\
\hline Trachea and main bronchus $(n=27)$ & $24(89)$ & $18(67)$ & $26(96)$ & 0.004 \\
\hline Upper lobes $(n=46)$ & $42(91)$ & $35(76)$ & $43(93)$ & 0.004 \\
\hline Right upper $(n=33)$ & $31(94)$ & $26(79)$ & $31(94)$ & 0.031 \\
\hline Left upper $(n=13)$ & $11(85)$ & $9(69)$ & $12(92)$ & 0.125 \\
\hline Right middle lobe $(n=9)$ & $7(78)$ & $5(56)$ & $9(100)$ & 0.063 \\
\hline Lower lobes $(n=12)$ & $9(75)$ & $8(67)$ & $11(92)$ & 0.125 \\
\hline Total $(n=94)$ & $82(87.2)$ & $66(70.2)$ & 89 (94.7) & $<0.001$ \\
\hline
\end{tabular}

${ }^{*}$ Fisher's exact test, ${ }^{\complement} \mathrm{CDT}$ versus TBNA + CDT.

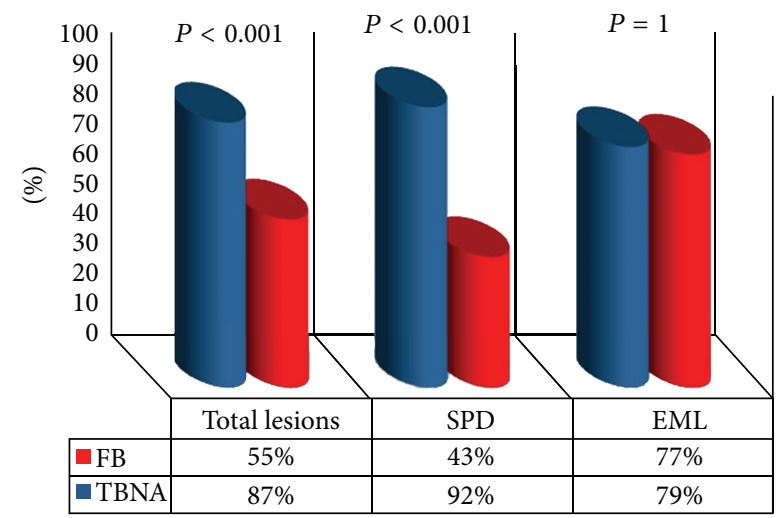

FIGURE 1: An illustration comparing the diagnostic yields of TBNA and forceps biopsy (FB) in the study cohort.

when occurred, stopped spontaneously. Bronchoscopic damage was not recorded.

\section{Discussion}

Despite the great advance in the improved sensitivity of TBNA for diagnosis and staging of lung cancer in the era of real-time endobronchial ultrasound-guided TBNA (EBUSTBNA) $[10,11]$, we believe that conventional TBNA is still underutilized for the diagnosis of endobronchial lung cancer. Only few studies reported the efficacy of conventional TBNA in the diagnosis of malignant endobronchial lesions [2-7]. In the current study, we aimed to report our experience with the diagnostic utility of TBNA in patients with visible malignant endobronchial lesions and to evaluate this utility in relation to different lesions' characteristics. To the best of our knowledge, this study is the first report to investigate the utility of TBNA in endobronchial malignant lesions in a developing country.

Our results demonstrated that the addition of TBNA to CDT improved the sensitivity of bronchoscopy from $70.2 \%$ to $94.7 \%$. When compared for different endobronchial lesion types, the addition of TBNA to CDT increased sensitivity in all lesion types, particularly in the peribronchial disease and submucosal-peribronchial disease groups. Despite that no statistical difference was observed in the EML group, TBNA identified five additional patients with lung cancer. These results are in agreement with those reported in previous studies $[2,6-8]$ and confirm that TBNA is a valuable diagnostic tool for malignant endobronchial lesions, particularly in PD. Minai and coworkers [1] stated that the yield of conventional procedures such as forceps biopsy and brushing tends to be much lower in submucosal and peribronchial disease than for exophytic lesions. Dasgupta et al. [2] demonstrated an increase in diagnostic yield in EML when TBNA was added to CDP; TBNA diagnosed lung cancer in four additional patients. They explained that by the potential ofTBNA to bypass surface necrosis and sample viable tumor from deep within the mass. Analysis of yield from individual procedures and their combinations (Table 2) again confirms the importance of TBNA especially for SPD lesions. The highest diagnostic yield by an individual procedure was obtained by TBNA (87.2\%) for the total number of lesions. Moreover, the rate of individual diagnostic yield was also the highest for TBNA for both EML (79.4\%) and SPD (91.7\%). More interestingly, the diagnostic yield from TBNA was far superior to the yield from combination of FB and brushing in total lesions and SPD. Thus, the use of TBNA alone or in combination with CDT would have diagnosed endobronchial lesions, particularly in SPD. These findings are consistent with those of Dasgupta et al. [2] who reported that TBNA had the highest diagnostic yield among individual procedures in EML, SPD, and total lesions. Similarly, Kaçar and coworkers [6], in their study of malignant endobronchial lesions in 95 patients, demonstrated that TBNA had the highest diagnostic yield among individual procedures in SPD and total lesions, but not in EML. The best combination of bronchoscopic procedures from the diagnostic point of view was questioned by many authors before. Dasgupta et al. [2] and Kaçar et al. [6] demonstrated that the optimal combination for diagnosis of both EML and SPD is forceps biopsy, TBNA, and brushing. On the other hand, Roth et al. [12] observed that the combination of only biopsy and TBNA had the highest diagnostic yield. In the current study, combination of TBNA with forceps biopsy and brushing provided the best diagnostic yield in EML, SPD, and total lesions. Our data showed that TBNA was exclusively diagnostic in $14 \%$ of total patients, $15 \%$ of patients with SPD, and $15 \%$ of patients with EML. These results, again confirm those shown in the literature. TBNA was the sole diagnostic technique in $20 \%$ [2], $17 \%$ [8], and $11 \%$ [6] of patients, respectively. On the other hand, we observed that FB and $\mathrm{BB}$ were exclusively diagnostic in $6 \%$ and $3 \%$ of patients; respectively, and all these patients had EML. These results are in agreement with the result of other authors. 
Forceps biopsy and $\mathrm{BB}$ were the sole diagnostic techniques in $3 \%$ and $6 \%$ [2], $13.8 \%$ and $1.5 \%$ [8], and $5 \%$ and $4 \%$ [6] of patients, respectively. Recent advances in bronchoscopic techniques had shown safety and higher diagnostic yield for endobronchial lesions using hot biopsy forceps or cryobiopsy in comparison to conventional biopsy [13-15]. Our data showed that bronchial washing was not exclusively diagnostic in situations in which all other procedures were negative. Several authors found that bronchial washing in relation to other sampling techniques for diagnosing bronchial tumours does not influence the diagnostic yield $[2,6,15]$. Notably, previous studies comparing the diagnostic yields of TBNA and forceps biopsy for malignant endobronchial lesions showed contradicting results. Roth et al. [12] and Lundgren et al. [16] reported a greater yield with forceps biopsy than TBNA (60\% versus $40 \%$ and $69 \%$ versus $46 \%$ ), respectively. However, reversed trends were shown by other authors [2-4]. Shure and Fedullo [4] showed an increased yield with TBNA (71\%) compared with forceps biopsy (55\%), similar to Buirski et al. [3] and Dasgupta et al. [2] who reported yields of $100 \%$ versus $43 \%$ and $85 \%$ versus $43 \%$, respectively. Our results (Figure 1) demonstrated a greater diagnostic yield of TBNA in comparison to forceps biopsy for all types of endobronchial lesions $(87.2 \%$ versus $55.3 \%$; $P<0.001)$. This difference was primarily resulting from differences in the SPD group.

When histologic subtypes are analyzed, the diagnostic sensitivity increased by addition of TBNA to CDT from $74.4 \%$ to $95 \%(P<0.001)$ and from $50 \%$ to $94 \%(P=$ 0.016 ) in NSCLC and SCLC, respectively. In NSCLC group, increased sensitivity was attributed to peribronchial disease. In SCLC group, there was no significant difference for any subtype of lesions within the group. The later observation could be due to small numbers of patients within each of the lesions' subtypes. This small number did not give enough power for statistical comparison. Our results are in accordance to those reported by Caglayan and coworkers [7]. They reported an increased sensitivity by the addition of TBNA to CDT in both NSCLC and SCLC from $61 \%$ to $87 \%$ and from $74 \%$ to $100 \%$, respectively. Similarly, Uskül et al. [17] in their study of 81 patients demonstrated that, by the addition of TBNA to CDT, diagnostic success was increased from $52 \%$ to $76 \%$ in the NSCLC group and from $81 \%$ to $95 \%$ in the SCLC group. Crush artifacts produced during biopsy sampling of small cell cancer may result in a negative result with the use of forceps biopsy. This situation was seen in two of our cases with EML in which TBNA was exclusively diagnostic. Jones et al. [18] have reported on the critical diagnostic value of TBNA in five patients with small cell cancer who demonstrated crush artifacts on non diagnostic forceps biopsy. Schenk and colleagues [19] had the best diagnostic rate with TBNA in SCLC (55\%), and the lowest rate was in adenocarcinoma (35\%). They attributed this difference to the increased cell count per volume in SCLC its more central location and that individual cells of small cell carcinomas are less adherent to one another than those of adenocarcinoma. The diagnostic utility of CDT and CDT plus TBNA according to the location of endobronchial lesions was addressed. Our results demonstrated that the addition of TBNA to CDT had increased the diagnostic yield irrespective of the location of the lesion, and this increase was significant in trachea and main bronchi and bronchi of the upper lobes. Interestingly, our results are consistent with those in the literature. Caglayan et al. [7] demonstrated increased sensitivity of CDT plus TBNA versus CDT in the trachea and main bronchi, upper lobes, and right middle lobe lesions. In another study, although the diagnostic success was increased in all localizations by the addition of TBNA to $\mathrm{CDT}$, significant increase was observed only for the lesions located at trachea and the main bronchi [17]. Anatomically, because of the acute angulation of the bronchoscope needed to reach them, FB may be difficult to perform in upper lobe lesions, particularly if they are distally located. No major complication due to TBNA of endobronchial lesions was reported in the literature $[2-4,6-8]$ which is consistent with our findings. Finally, despite that cost effectiveness was not carried out, the current study might have important implications. Being a safe and cost-effective, TBNA is a useful diagnostic tool in the hands of bronchoscopists in developing countries. This will avoid unnecessary, repeat, and/or expensive procedures, thus improving the outcomes of bronchoscopy for lung cancer.

\section{Conclusion}

Our results confirm previous data that TBNA is a safe and valuable diagnostic technique for malignant endobronchial lesions. Utility of TBNA is evident as a complementary tool for CDT in the diagnosis of malignant endobronchial lesions, particularly in peribronchial disease. Addition of TBNA to CDT improves the diagnostic yield of bronchoscopy, in both NSCLC and SCLC and in all bronchoscopic locations; particularly in central and upper lobar lesions.

\section{Conflict of Interests}

All authors do not have potential conflict of interests.

\section{Disclosure}

No portion of this work has been published previously or is under consideration for publication elsewhere and is an original paper. The authors declare no financial support for this work.

\section{References}

[1] O. A. Minai, A. Dasgupta, and A. C. Mehta, "Transbronchial needle aspiration of central and peripheral lesions," in Interventional Bronchoscopy, C. T. Bolliger and P. N. Mathur, Eds., vol. 30, pp. 66-79, Karger, Basel, Switzerland, 2000.

[2] A. Dasgupta, P. Jain, O. A. Minai et al., "Utility of transbronchial needle aspiration in the diagnosis of endobronchial lesions," Chest, vol. 115, pp. 1237-1241, 1999.

[3] G. Buirski, P. M. A. Calverley, and N. J. Douglas, "Bronchial needle aspiration in the diagnosis of bronchial carcinoma," Thorax, vol. 36, no. 7, pp. 508-511, 1981.

[4] D. Shure and P. F. Fedullo, "Transbronchial needle aspiration in the diagnosis of submucosal and peribronchial bronchogenic carcinoma," Chest, vol. 88, no. 1, pp. 49-51, 1985. 
[5] S. Bilaceroglu, O. Gunel, U. Cagirici, and K. Perim, "Comparison of endobronchial needle aspiration with forceps and brush biopsies in the diagnosis of endobronchial lung cancer," Monaldi Archives for Chest Disease, vol. 52, no. 1, pp. 13-17, 1997.

[6] N. Kaçar, F. Tuksavul, Ö. Edipoğlu, S. Ermete, and S. Z. Güčlü, "Effectiveness of transbronchial needle aspiration in the diagnosis of exophytic endobronchial lesions and submucosal/peribronchial diseases of the lung," Lung Cancer, vol. 50, no. 2, pp. 221-226, 2005.

[7] B. Caglayan, U. A. Akturk, A. Fidan et al., "Transbronchial needle aspiration in the diagnosis of endobronchial malignant lesions: a 3-year experience," Chest, vol. 128, no. 2, pp. 704-708, 2005.

[8] J. A. Gullón, R. Fernández, A. Medina et al., "Transbronchial needle aspiration in bronchogenic carcinoma with visible lesions: diagnostic yield and cost," Archivos de Bronconeumologia, vol. 39, no. 11, pp. 496-500, 2003.

[9] K.-P. Wang, "Transbronchial needle aspiration and percutaneous needle aspiration for staging and diagnosis of lung cancer," Clinics in Chest Medicine, vol. 16, no. 3, pp. 535-552, 1995.

[10] L. Kokkonouzis, A. S. Strimpakos, L. Lampaditis, S. Tsimpoukis, and K. N. Syrigos, "The role of endobronchial ultrasound in lung cancer diagnosis and staging: a comprehensive review," Clinical Lung Cancer, vol. 13, pp. 408-415, 2012.

[11] S. Mohamed, K. Yasufuku, T. Nakajima et al., "Analysis of cell cycle-related proteins in mediastinal lymph nodes of patients with N2-NSCLC obtained by EBUS-TBNA: relevance to chemotherapy response," Thorax, vol. 63, no. 7, pp. 642-647, 2008.

[12] K. Roth, J. A. Hardie, A. H. Andreassen, F. Leh, and T. M. L. Eagan, "Predictors of diagnostic yield in bronchoscopy: a retrospective cohort study comparing different combinations of sampling techniques," BMC Pulmonary Medicine, vol. 8, article 2, 2008.

[13] A. Khan, A. N. Aggarwal, R. Agarwal, A. Bal, and D. Gupta, "A randomized controlled trial of electrocoagulation-enabled biopsy versus conventional biopsy in the diagnosis of endobronchial lesions," Respiration, vol. 81, no. 2, pp. 129-133, 2011.

[14] C. Schumann, J. Hetzel, A. J. Babiak et al., "Cryoprobe biopsy increases the diagnostic yield in endobronchial tumor lesions," Journal of Thoracic and Cardiovascular Surgery, vol. 140, no. 2, pp. 417-421, 2010.

[15] J. Dionísio, "Diagnostic flexible bronchoscopy and accessory techniques," Revista Portuguesa de Pneumologia, vol. 18, no. 2, pp. 99-106, 2012.

[16] R. Lundgren, F. Bergman, and T. Angstrom, "Comparison of transbronchial fine needle aspiration biopsy, aspiration of bronchial secretion, bronchial washing, brush biopsy and forceps biopsy in the diagnosis of lung cancer," European Journal of Respiratory Diseases, vol. 64, no. 5, pp. 378-385, 1983.

[17] B. T. Uskül, H. Türker, A. Melikoğlu, A. Yilmaz, S. Boğa, and C. Ulman, "Value of transbronchial needle aspiration in the diagnosis of endobronchial malignant lesions," Tüberküloz ve Toraks, vol. 55, pp. 259-265, 2007.

[18] D. F. Jones, R. Chin Jr., J. O. Cappellari, and E. F. Haponik, "Endobronchial needle aspiration in the diagnosis of small-cell carcinoma," Chest, vol. 105, no. 4, pp. 1151-1154, 1994.

[19] D. A. Schenk, C. L. Bryan, J. H. Bower, and D. L. Myers, “Transbronchial needle aspiration in the diagnosis of bronchogenic carcinoma," Chest, vol. 92, no. 1, pp. 83-85, 1987. 


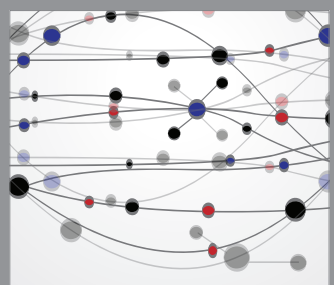

The Scientific World Journal
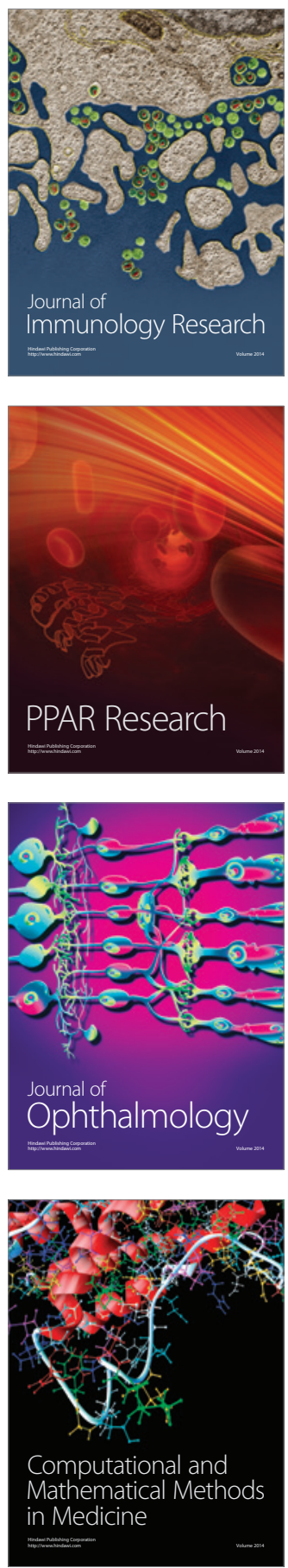

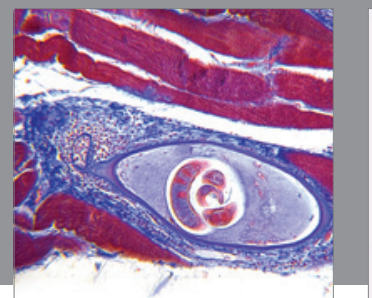

Gastroenterology

Research and Practice
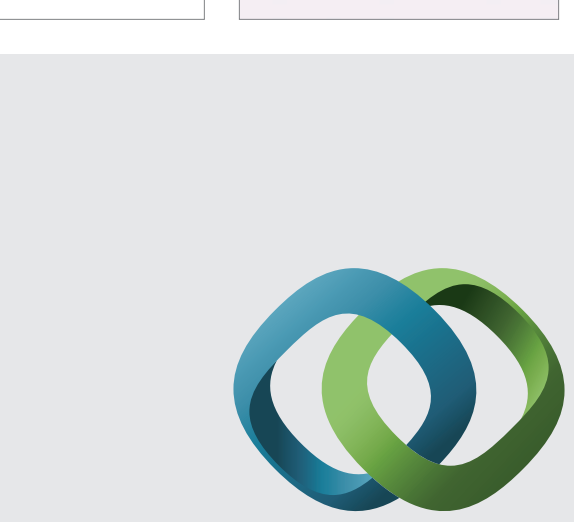

\section{Hindawi}

Submit your manuscripts at

http://www.hindawi.com
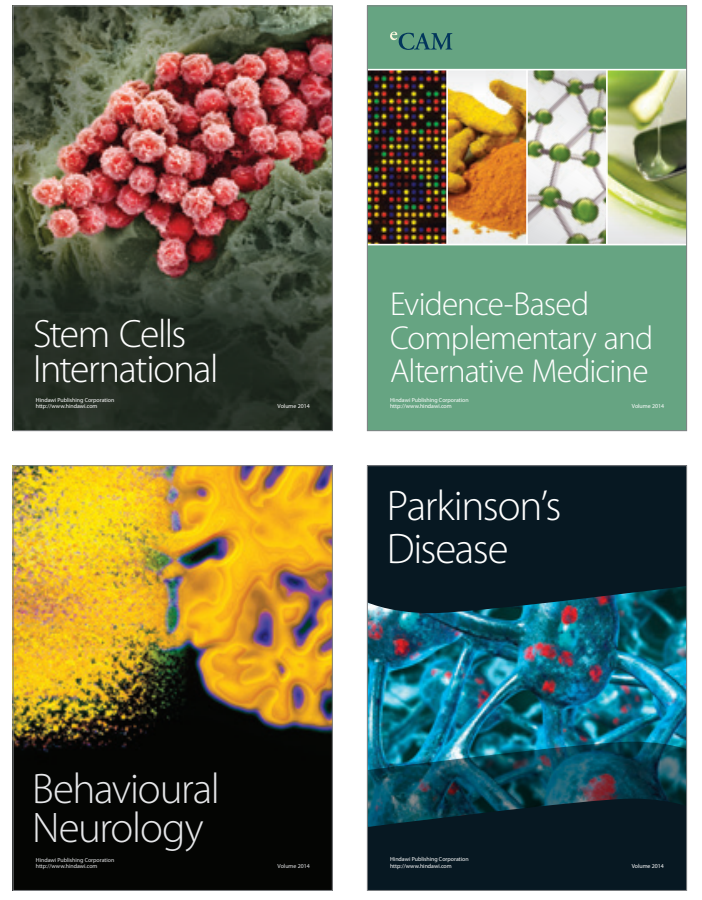
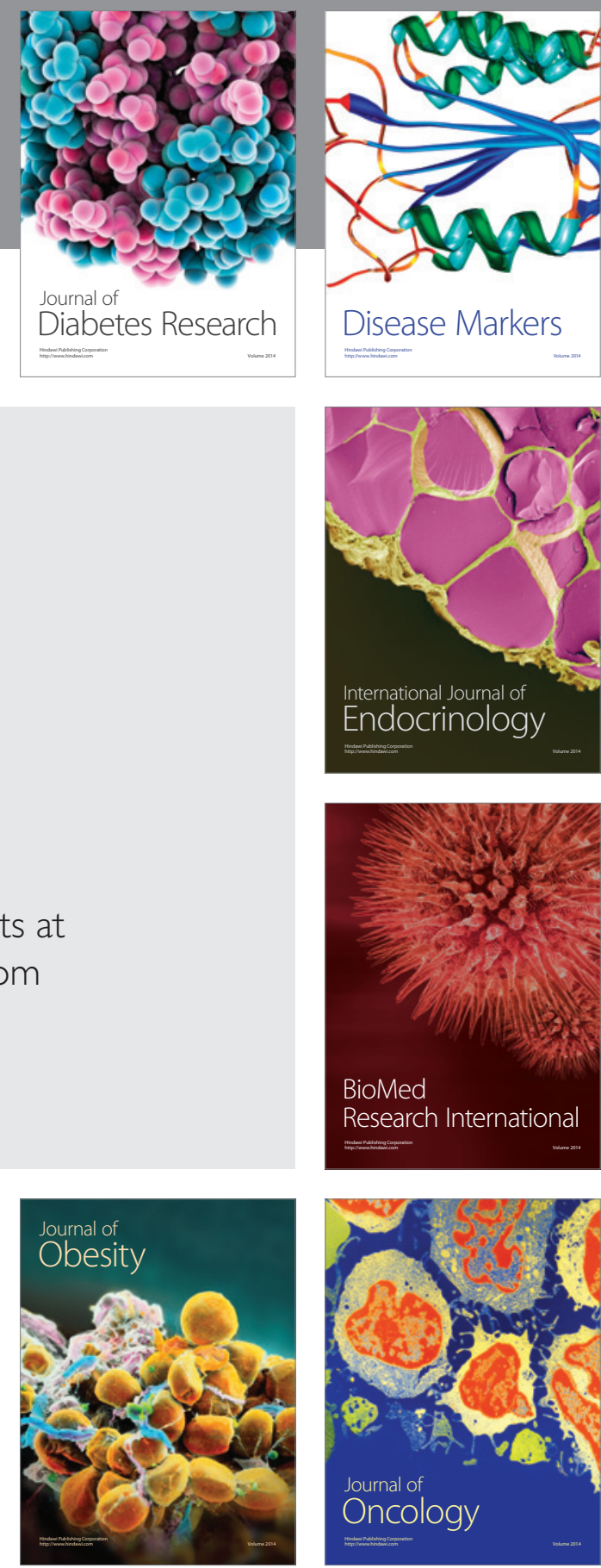

Disease Markers
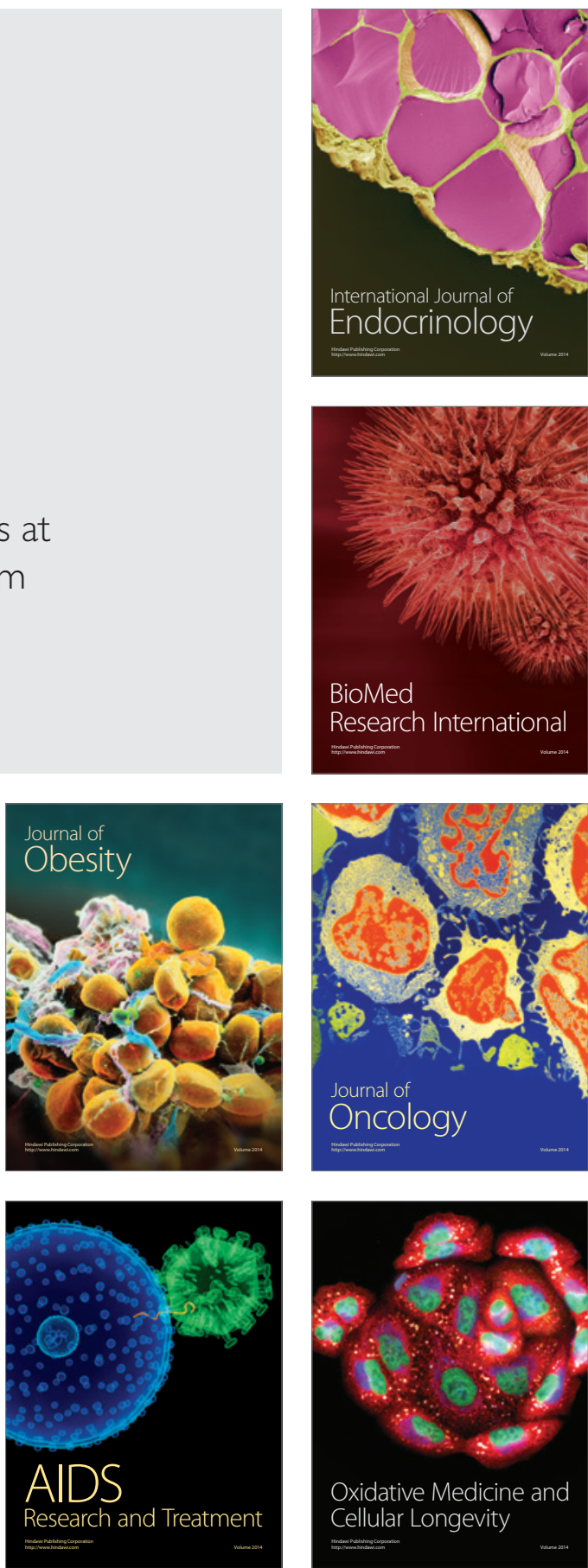